\title{
Pluronic F-127 and Pluronic Lecithin Organogel (PLO): Main Features and their Applications in Topical and Transdermal Administration of Drugs
}

\author{
Hugo Almeida, Maria Helena Amaral, Paulo Lobão, José Manuel Sousa Lobo. \\ Pharmaceutical Technology Laboratory, Drug Sciences Department, Faculty of Pharmacy, University of Porto, \\ Portugal.
}

Received, Septmber 25, 2012; Accepted, October 10, 2012; Published, October 25, 2012.

\begin{abstract}
Topical drug treatment aims at providing high concentrations of drugs at the site of application so as to avoid adverse systemic effects associated with oral administration. Smart polymers, or stimuli-responsive polymers, are able to respond to a stimulus by showing physical or chemical changes in their behaviour as, for example, the delivery of the drug carried by them. The thermo-responsive nature of Pluronic ${ }^{\circledR}$ F-127 (Basf, Ludwigshafen, Germany) makes it an excellent candidate for the delivery of drugs at various application sites. In recent years, PF-127, and later, Pluronic lecithin organogels (PLO), have attracted particular interest in the design of dermal and transdermal delivery systems with a view to promoting, improving or retarding drug permeation through the skin, bearing in mind that for topical delivery systems, accumulation in the skin with minimal permeation is desired, while for systemic delivery, the opposite behaviour is preferred.

In this review, we discuss the properties and characteristics of PF-127 and Pluronic lecithin organogels (PLO), and present many examples and advantages of the application of these polymeric systems in topical and transdermal administration of drugs.
\end{abstract}

This article is open to POST-PUBLICATION REVIEW. Registered readers (see "For Readers") may comment by clicking on ABSTRACT on the issue's contents page.

\section{INTRODUCTION}

Smart polymers, or stimuli-responsive polymers, have been incorporated into the vanguard of drug administration technology as they demonstrate active responses to small changes in the surrounding environment (physical stimulus temperature, ultrasound, light, mechanical stress; chemical stimulus - $\mathrm{pH}$ and ionic strength; biological stimulus - enzymes and biomolecules), which translate into physical or chemical reversible changes in their behaviour as, for example, the delivery of the drug carried by them (1-7).

The main advantages that the addition of smart polymers to drug molecules provide include the ability to administer an efficient concentration of a certain drug at the right time and location, a reduction in adverse systemic reactions, and an increase the patient's adherence to the therapeutic regimen, thereby also allowing a reduction in the drug dose and, consequently, the costs (8).

The development of in situ gel systems has received considerable attention over the past few years (9). It is well-known that gels are swollen networks possessing both the cohesive properties of solids, and the diffusive transport characteristics of liquids. Over the last few decades, gels formed from natural, semisynthetic or synthetic polymers have been confirmed as vehicles for different types of pharmaceutical applications (10).

Generally, hydrogel bases can be easily washed off, but they adhere well to skin or mucous membranes or skin, wet with secreting fluid and thus these systems are usually applied to injured skin and also to eyes (10). The in situ gelforming polymeric formulations offer several advantages such as sustained and prolonged action in comparison to conventional drug delivery systems. From a manufacturing point of view, the production of such devices is less complex and thus lowers the investment and manufacturing costs (9).

Block copolymers are widely used industrially in the solid and rubbery states. They are used as thermoplastic elastomers, with applications in impact modification and pressure sensitive adhesion (11). The use of hydrosoluble vehicles of high viscosity, such as hydrophilic gels, is one of many strategies to obtain a controlled delivery and represents an area very

Corresponding Author: Hugo Almeida, Pharmaceutical Technology Laboratory, Faculty of Pharmacy, University of Porto, Rua Jorge Viterbo Ferreira, Porto, Portugal; Email: hperas5@hotmail.com 
important in scientific research (12). Reversible gels refer to those that have the capacity to make, break, and modify the bonds responsible for holding the network together $(11,13)$.

A series of amphiphilic block copolymers have been reported that have temperatureresponsive micellisation behaviour and which form hydrogels when above a critical gelation temperature (14).

\section{POLOXAMERS}

\section{General characteristics}

Poloxamers are non-ionic polymers of polyoxyethylene-polyoxypropylene-

polyoxyethylene $\left(\mathrm{PEO}_{\mathrm{n}}-\mathrm{PPO}_{\mathrm{n}}-\mathrm{PEO}_{\mathrm{n}}\right)$, which have many pharmaceutical uses (Figure 1) $(9,13,15-$ 18). Block copolymers, based on PEO-PPO sequences (ABA-type triblock copolymers composed of PEO (A) and PPO units (B)), are a family of commercially available triblock copolymers which have the following trade names: Pluronics, Poloxamers or Tetronics (1821).

Poloxamers, like other surfactants, when dispersed in the liquid, at low concentrations, exist individually as monomolecular micelles. But as the concentration of the poloxamer in the system increases, this results in the formation of multimolecular aggregates (22). Polypropylene oxide (PPO) forms central hydrophobic core wherein methyl groups interact via van der Waals forces with substance undergoing solubilisation. However, water solubility is believed to be due to the polyethylene oxide (PEO) block by hydrogen bonding interactions of ether oxygen with water molecules. Due to these interactions, Pluronics are readily soluble in non-polar organic solvents and established themselves in the formulation of dosage forms (23). Pluronics show different aggregate forms depending on the molecular weight, block sizes, solvent composition, and temperature.

Aqueous solutions of Pluronics in the presence of acids, alkalis, and metal ions are very stable. The Pluronic triblock copolymers are available in various grades differing in molecular weights and physical forms. Depending upon the physical designation the grades are assigned, as $\mathrm{F}$ for flakes, $\mathrm{P}$ for paste, or $\mathrm{L}$ for liquid. The polaxamers normally used are: 188 (F-68), 237 (F-87), 338 (F-108) and 407 (F-127) (9).

These polymers are composed of white granules that are soluble in water and have no odor or taste. They present a sol-gel transition phase below or near the physiological body temperature and a gel-sol transition at around 50 ${ }^{\circ} \mathrm{C}$ in relatively highly concentrations $(2,9,13,15,16,24-26)$.

Pluronics and Tetronics are used as thermoreversible gels with some examples having been approved by the FDA for applications which include as food additives, pharmaceutical ingredients and agricultural products, drug delivery carriers and in injectable systems for tissue engineering processes (19).

\section{PLURONIC F-127}

\section{Characteristics and properties}

Pluronic ${ }^{\circledR}$ F-127 (Basf, Ludwigshafen, Germany), also known as poloxamer 407 (P407), (copolymer polyoxyethylene $_{106}$-polyoxypropylene ${ }_{70^{-}}$

polyoxyethylene ${ }_{106}$ ) contains about $70 \%$ ethylene oxide, which contributes to its hydrophilicity (25). PF-127 is a copolymer with a weight of 12,000 daltons, a PEO/PPO ratio of 2:1, and is non-toxic, with low viscosity below $4{ }^{\circ} \mathrm{C}$ and which forms a semi-solid gel at body temperature (25-29). PF127 is more soluble in cold water than in hot water due to the increased solvation and hydrogen linkages at low temperatures $(2,11)$.
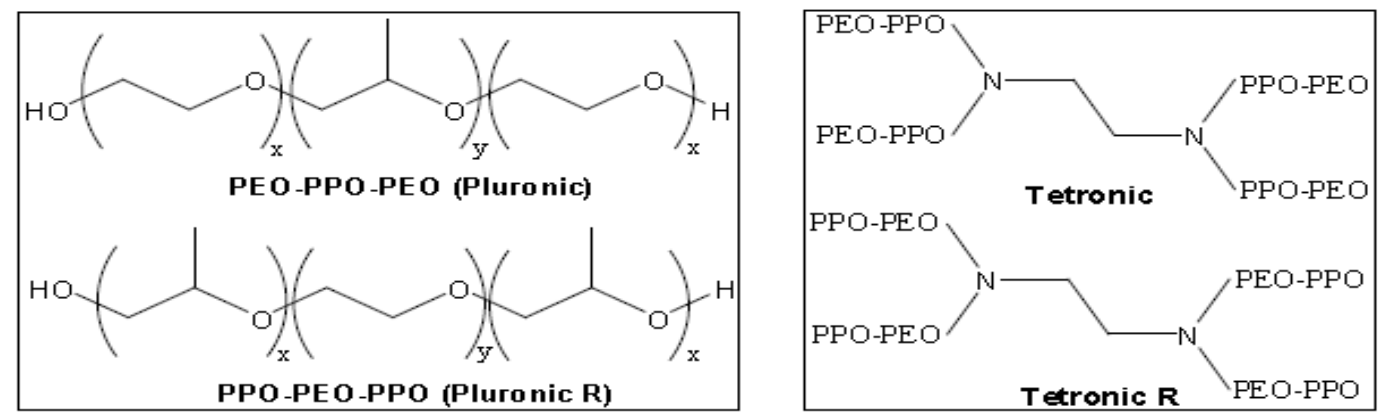

Figure 1. Schematic structure of polymers with amphiphilic balance (19). 
The aqueous solutions of PF-127 at concentrations between $20-30 \% \quad(\mathrm{~m} / \mathrm{m})$, reversibly turn into gels at a certain temperatures, i.e. they become more liquid at lower temperatures $\left(4-5{ }^{\circ} \mathrm{C}\right)$ and turn into gels at body temperature (this transformation is reversible and thus solutions return to a liquid state at low temperatures) $\quad(11,13,16,25,27,28)$. This thermogelling results from interactions between the different molecules of Pluronic. The increase in temperature modifies the hydration spheres around the hydrophobic units, which in turn induces higher interactions between these different units $(2,30)$.

In other words, with the increase in temperature of a PF-127 aqueous solution, the PPO block tends to dehydrate and form a core with an outer shell of hydrated PEO chains that aggregate into spherical micelles. The micellar structure of this copolymer in an aqueous environment can be used for incorporation of hydrophilic and hydrophobic drugs, and prolongs drug release $(21,29)$. This characteristic, in addition its low toxicity, biocompatibility with cells and body fluids, and weak immunogenic properties, means PF-127 is a commonly used polymer in drug delivery systems $(10,13,15,24,25,29,31)$.

\section{Rheological characteristics}

The gelation temperature of a polymer depends on polymer composition and solution concentration $(19,32)$. Studies have also shown that the drug diffusion coefficient in the gel decreases as PF127 content increases, coinciding with an increase in gel viscosity. This led the authors to propose that drug release rates are determined by gel viscosity (33). The drug delivery from polymer PF-127 occurs by diffusion and dissolution through the gel that is formed on the area where it was administrated (33). PF-127, at low percentages, has a complete dissolution in water in 4 hours and this feature can be used to prepare pharmaceutical formulas that act in short timespans after administration (short-term therapy). Ricci et al. (17) meanwhile showed that the diffusion coefficient of a drug decreases when the concentration of this polymer is increased. A $25-40 \%$ aqueous solution of this material will therefore form a gel at body temperature, and drug release from such a gel occurs over a period of up to one week (9). These authors thus also concluded that the time of dissolution increases when drug diffusion through the gel matrix is decreased.
There is therefore a relationship between viscosity and temperature in these gels, with the slope dependent upon PF-127 concentration. This phenomenon was explained on the basis of a previously reported observation that $\mathrm{PF}-127$ micelles in aqueous solution undergo a thermallyinduced swelling and desolvation (11).

It was reported by many researchers that various salts, surfactants, polymers, cosolvents and other additives added have marked effects on micellisation, clouding, and solubilisation behaviour of Pluronic solutions. Since almost all pharmaceutical PF-127 gels are formulated with buffer salts, and may contain the salt form of a drug, some authors felt it was important to further investigate the effect of common inorganic salts on these transition temperatures (34). Appropriate salts could potentially be used to tailor the temperatures at which these transitions in PF-127 gels occur, in order to design unique thermoresponsive drug delivery systems. The alteration of these transition temperatures however may also influence the diffusion of the drug in the gel and, hence, release rates (34).

Pandit and Kisaka (34) concluded in their studies that all salts studied $\left(\mathrm{NaCl}, \mathrm{Na}_{2} \mathrm{SO}_{4}\right.$, $\mathrm{Na}_{3} \mathrm{PO}_{4}, \mathrm{CaCl}_{2}, \mathrm{MgSO}_{4}$ and $\left.\mathrm{Al}_{2}\left(\mathrm{SO}_{4}\right)_{3}\right)$ lower the transition temperature. The degree of lowering is proportional to salt concentration, and can be ascribed to salting-out effects. This behaviour parallels the effect of these salts on the temperature-solubility behaviour of aqueous poly(oxyethylene) solutions. The release rate and diffusion coefficient of the drug tested (propranolol) was significantly reduced when $\mathrm{NaCl}, \mathrm{Na}_{2} \mathrm{SO}_{4}, \mathrm{NaH}_{2} \mathrm{PO}_{4}, \mathrm{MgSO}_{4}$ and $\mathrm{CaCl}_{2}$ were added to the gels (the magnitude of the effect on release rate depends on the nature and concentration of the salt) (35).

With the same objective, Ricci et al. (17) showed that the incorporation of organic solvents or salts (sodium chloride or PEG 400 hydrophilic substances) changes the sol-gel transition temperature of PF-127, increasing also the drug delivery rate (lidocaine hydrochloride). In addition, gel viscosity can be increased by raising the polymer concentration, and hydrophilicity can be increased by adding salts (the presence of $\mathrm{NaCl}$ or PEG 400 increases drug release rates). Thus, PF-127 can be used to increase the duration of action of lidocaine, increasing the drug efficiency and decreasing the side effects (17). The viscosity of PF-127 gel in water was slightly lower than in presence of sodium chloride.

The addition of isotonic agents (examples: mannitol, sorbitol and sodium chloride) or 
viscosity enhancing agents (examples: hydroxypropylmethyl cellulose, methyl cellulose, carboxymethylcellulose sodium) promotes the increase in viscosity of PF-127 formulations and contributes to the decreased rate of drug release (30).

On the other hand, we know that the gelation temperature of a specific polymeric system is determined by the hydrophilic-hydrophobic balance existing in the polymer (32). This balance can be modulated by incorporating different side chains with hydrophilic or hydrophobic segments. By grafting poly(acrylic acid) (PAA) onto the poloxamer backbone in a one-step reaction via radical polymerisation of acrylic acid (AA) in the presence of poloxamer, the sol-gel transition occurs at a lower concentration than for poloxamer alone as PAA forms physical crosslinking points at low concentrations. The graft copolymer of poloxamers with PAA has several advantages; thermally reversible gelling behaviour over a wide $\mathrm{pH}$ range, no phase separation, and bioadhesive properties, all combined in a single molecule (14). In the case of injection of poloxamer into the body, the hydrogel structure of this polymer was eroded from its surface into soluble unimers within one day. More durable and biocompatible block copolymers that undergo micelle aggregation and packing were obtained when the PPO block was replaced with poly(L-lactic acid) and more recently with poly(DL-lactic acid-co-glycolic acid) (PLGA), which contains a biodegradable ester group in its backbone (14).

Anti-inflammatory drugs like indomethacin and naproxen, which are hydrophobic, cause a decrease in micellar size. These drugs also cause decreases in aggregation numbers leading to decreases in the number of micelles (36).

\section{Enhancement of mucosal absorption}

There are some drugs, like peptides and proteins, that are very difficult to deliver by conventional methods through the skin because they are polar, charged or have a large molecular weight. In addition, unlike most small drug molecules, some drugs and peptides do not cross the mucosal membrane efficiently. This low mucosal absorption can be attributed to poor membrane permeability due to molecular size, lack of lipophilicity, or enzymatic degradation (9). To overcome these problems, one of the most frequently used approaches is the use of absorption enhancers. These act by one, or a combination, of the following mechanisms: alteration of properties of the mucosal layer, opening of tight junctions between epithelial cells, reverse micelle formation between membranes, and by increasing the membrane fluidity.

However, the use of enhancing-transport technology, such as iontophoresis, in combination with chemical enhancers provides the opportunity to deliver these kinds of drugs through the skin by increasing permeation (11). Various types of penetration enhancers have been evaluated for organic drugs including surfactants, bile salts, chelators, fatty acid salts, phospholipids, glycyrrhetinic acid derivatives, cyclodextrins and glycols $(9,37,38)$.

Morishita et al. (39) studied the influence of the addition of the unsaturated fatty acids (such as oleic acid (18:1), eicosapentaenoic acid (20:5) or docosahexaenoic acid (22:6)) on the spreadability and hypoglycemic effect of insulin following buccal administration of the gel formulations in normal rats. All the formulations with unsaturated fatty acids exhibited significantly lower spreadability than the control PF-127 formulation. The increase in the viscosity might have therefore contributed to the decrease in insulin release seen.

Bentley et al. (40) concluded in their studies that the presence of lecithin in a poloxamer gel improved the characteristics for topical drug delivery. The addition of lecithin (a permeation enhancer) increased the thixotropy, apparent viscosity and the gelation temperature of the gels. The presence of lecithin in PF-127 gels however decreased the flux of a lipophilic drug through the skin, increasing its skin retention.

Abdel-Mottaleb et al. (10) studied the topical administration of fluconazole in PF-127 gels with addition of different additives. The additives used were PEG 400, glycerol, tween 80 and cetrimide. Only the formulations with PEG 400 or glycerol increased the viscosity of the product. Tween 80 and cetrimide, on the other hand, caused a significant decrease in the gel viscosity. All the additives however produced a significant enhancement in the release of fluconazole in comparison with the additive free base, with the highest release being obtained from the formula containing glycerol.

Gonjari et al. (41) showed that the addition of penetration enhancers (sodium glycocholate, EDTA or transcutol) in transnasal formulations of PF-127 increased the nasal absorption of sumatriptan succinate. It was also shown that such absorption enhancers increased the gelation temperature of the PF-127 base.

Fitzgerald et al. (42) demonstrated that the combination of glycerol and PF-127 produced additive enhancements in naproxen accumulation inside SCC-25 cells (derived from oral mucosa). 
Compared with untreated control cells, naproxen content was $74 \%$ higher in cells treated with 5\% glycerol and $0.05 \%$ PF-127. Individually, 5\% glycerol and $0.05 \%$ PF-127 produced enhancements of approximately $48 \%$ and $21 \%$, respectively. PF-127 enhances the solubilisation and cell permeability of lipophilic drugs, and is also capable of altering the properties of the plasma membrane.

These gels however, possess poor bioadhesiveness and high permeability to water, which limited their application as a thermoresponsive matrix. To resolve this problem, Yuan et al. (43) developed a thermosensitive and mucoadhesive rectal in situ gel of nimesulide (NM) by using mucoadhesive polymers such as sodium alginate $(\mathrm{Alg}-\mathrm{Na})$ and HPMC. These gels were prepared by addition of mucoadhesive polymers $(0.5 \%)$ to the formulations of thermosensitive gelling solution containing PF-127 (18\%) and nimesulide (2\%). Polyethylene glycol (PEG) was used to modify gelation temperature and drug release properties. Gelation temperature was significantly increased with incorporation of nimesulide $(2 \%)$ in the poloxamer solution, while the addition of the mucoadhesive polymers played an opposite role on gelation temperature. The addition of PEG polymers increased the gelation temperature and the drug release rate.

Nirmal et al. (9) used PF-127 as an in situ gelforming polymer together with mucoadhesive polymers such as Carbopol 934 and hydroxylpropylmethylcellulose to ensure long residence time at the application site. With the same objective, Shin et al. (44) used two polymers, Carbopol 934 and PF-127 with different enhancer bile salts, glycols and nonionic surfactants (examples: sodium cholate, sodium taurodeoxycholate, sodium deoxycholate, tetraethylene glycol, diethylene glycol, polyoxyethylene 2-stearyl ether, polyoxyethylene 23-lauryl ether, polyoxyethylene 2-oleyl ether) to enhance permeation of triamcinolone acetonide through buccal mucosa. Among the enhancers used, sodium deoxycholate showed the best enhancement effects.

\section{Applications of PF-127 gels in pharmaceutical formulations}

PF-127 (poloxamer 407) can be used as a carrier for several routes of administration, including oral, subcutaneous, intranasal, vaginal, rectal, ocular and parenteral $(13,45)$. In the last few years, PF-127 has also had an important role in dermal and transdermal drug delivery systems (13,31).

Some studies have been carried out to test incorporation of PF-127 in short-term therapies such as, for example, fertility control, pain management and infections treatment (46). This kind of polymer has been already tested for external uses (local and sustained delivery) of anticancer and anti-inflammatory drugs $(19,31,46)$. It has been also tested in ophthalmology using pilocarpine $(2,11)$. There have been reported studies related to parenteral formulations (intramuscular and subcutaneous) with interleukin-2 and antibiotics (17). In this same manner, this poloxamer has been successfully tested to carry peptides and proteins (insulin, urease and growth factors), presenting a sustained delivery profile for several hours $(26,45)$.

\section{Topical and dermal applications}

Topical drug treatment aims at providing high concentrations of the drug at the site of application so as to avoid adverse systemic effects associated with oral administration of the drug (47). The delivery of drugs onto the skin, for example, is recognised as an effective means of therapy for local dermatologic diseases (10).

In recent years PF-127 has attracted particular interest in the design of dermal and transdermal delivery systems, with a view to promoting, improving or retarding drug permeation through the skin, bearing in mind that for topical delivery systems, accumulation in the skin with minimal permeation is desired, while for systemic delivery, the opposite behaviour is preferred $(11,15)$. In particular, focus has been on the development of topical/dermal formulations containing analgesic or anti-inflammatory drugs due to the fact that the potential for the delivery of these drugs through the skin, for local pain and inflammations at low doses, is attractive. However, in many cases penetration enhancers may also have to be included in the topical/dermal formulations as otherwise only small amounts of drug pass through the skin (11).

Table 1 shows some of the many examples of the use of PF-127 in pharmaceutical systems over time. 
Table 1. Examples of the use of Pluronic F-127 (PF-127) in pharmaceutical systems.

\begin{tabular}{l} 
Pharmaceutical \\
Formulations \\
\hline Topical administration of \\
anticancer agents (5- \\
flurouracil and adriamycin) \\
formulated in PF-127 gels. \\
Percutaneous administration \\
of indomethacin using a rat \\
model. \\
Transdermal gel comprised \\
of ketoprofen; PF-127; and \\
one or more agents selected \\
from: ethyl alcohol, \\
isopropyl alcohol, propylene \\
glycol, polyethylene glycol \\
and glycerin; as well as one \\
or more agents selected \\
from the group of: lauric \\
acid, oleic acid, capric acid, \\
myristic acid, lauryl alcohol \\
and menthol; plus either \\
water or a buffer solution. \\
and
\end{tabular}

Percutaneous administration of fentanyl formulated in PF-127 gels (in vitro and in vivo studies).

The effects of PF-127 on the permeability of several weak acids and bases through bilayer lipid membranes.

PF-127 hydrogels with piroxicam, and the effects of different non-ionic surfactants as permeation enhancers of the skin (polyoxyethylene-23-lauryl

ether, polyoxyethylene-2oleyl ether and polyoxyethylene-2-stearyl ether).

PF-127 hydrogels with capsaicin and nonivamide (in vitro and in vivo studies).

In vitro percutaneous absorption of nonivamide from gels of various polymers (PF-127, chitosan and carboxymethylcellulose)
Results

References

With increasing concentrations of PF-127 in the vehicle, a corresponding decrease in the apparent release rate of the anticancer agent occurred.

The apparent release rate increased with increasing temperature from 30 to $44{ }^{\circ} \mathrm{C}$.

PF-127 is a good vehicle for percutaneous absorption.

The addition of isopropyl myristate or $(+)$ - limonene improved percutaneous absorption (particularly when the gel was applied using an occlusive dressing technique).

The gels possess prolonged anti-inflammatory and analgesic activities, and physicochemical stability, with fewer systemic side effects and gastric irritation, compared to oral administration.

PF-127 has the potential to increase the therapeutic efficacy of fentanyl citrate or other lipophilic drugs by prolonging percutaneous input into the systemic circulation of rabbits.

PF-127 facilitated the permeation of comparatively large molecules (such as 2-n-undecylmalonic acid and doxorubicin) across lipid bilayers, while the permeation of small solutes (such as ammonium and acetic acid) remained unaffected.

Pluronics also accelerate the translocation of large hydrophobic anions (tetraphenylborate).

Poloxamer gels containing piroxicam, and including surfactants as enhancers, are good preparations to promote the percutaneous absorption of drugs.

The different non-ionic surfactants give rise to increases in the permeation of the skin.

Among the various non-ionic surfactants tested, polyoxyethylene-2oleyl ether showed the highest enhancement effects.

The incorporation of PF-127 polymer into hydrogels resulted in a retarded release of capsaicin.

The in vitro permeation of capsaicin from hydrogels depends on the physicochemical nature and the concentration of the polymer used.

The permeation of nonivamide was retarded in later stages of in vitro application.

The incorporation of PF-127 polymer into hydrogels resulted in retarded release of nonivamide.

Chitosan and carboxymethylcellulose hydrogels produced higher levels of in vitro nonivamide permeation and skin distribution.

The in vivo effects of nonivamide on skin perturbation and vasodilation were found to differ depending on dose and duration after topical 
using Wistar rat as an application.

animal model.

PF-127 gel formulated with ceftiofur. PF-127 (25-35\% $\mathrm{w} / \mathrm{v})$ was tested alone or with polyvinyl pyrrolidone (PVP),

carboxymethylcellulose

(CMC), or hydroxylpropyl

methylcellulose

(HPMC) as an additive.

PF-127 gel formulated with indomethacin

(hexylene glycol (HG) or polyethylene glycol 300 (PEG) as solvents). Tween 80 and PVP were added as excipients.

PF-127 gel formulated with insulin (ex vivo and in vivo skin permeation in rats with chemical enhancer and/or iontophoresis).

Transdermal timolol delivery from a PF-127 gel.

PF-127 and hydroxypropyl methylcellulose (HPMC) formulated pranoprofen.

Preparation and evaluation of pranoprofen gel for percutaneous administration.

Transdermal honokiol
formulation based on PF127 copolymer.

In vitro release of piroxicam in microemulsion formulations from different pharmaceutical topical preparations including
The release of ceftiofur is controlled by dissolution of PF-127.

An increase in PF-127 content from 25 to $35 \%$ resulted in a decrease in the rate of ceftiofur release.

PVP, CMC, and HPMC in the gel decreased the rate of release of ceftiofur to some extent.

Results indicate that the excipients influence the physical characteristics of the gels. The optimum concentration for gels manifesting as strength of gel was $20 \%$ PEG in combination with $1 \%$ PVP, which had the highest viscosity and yield value at a low shear rate.

Increasing the amount of HG or PEG gave a more viscous gel, with the exception of the $24 \% \mathrm{w} / \mathrm{w}$ HG gels which turned a jelly with or without either Tween or PVP.

Linoleic acid and menthone, in combination with iontophoresis, showed a synergistic enhancement of insulin permeation.

Iontophoresis, either alone or in combination with linoleic acid, produced a reduction in PGL (plasma glucose levels) to the extent of $36-40 \%$.

PF-127 gel and an artificial membrane are used to regulate the timolol delivery through pig stratum corneum.

At low PF-127 concentrations and for large pore-size membranes (Polyflux, PES-30), the stratum corneum mainly controls timolol delivery.

At high PF-127 concentrations and for small pore-size membranes (NF-PES-10), the contribution of the device (gel plus artificial membrane) to the timolol delivery is significant.

To increase drug permeation, several types of penetration enhancers such as ethylene glycols, propylene glycols, glycerides, non-ionic surfactants, and fatty acids, were incorporated in the gel formulation.

Among the various enhancers used, propylene glycol monolaurate showed the highest enhancement effects.

The results of this study suggest that development of a topical gel formulation of pranoprofen with an enhancer is feasible.

Various penetration enhancers, such as non-ionic surfactants and fatty acids, were incorporated into the gel formulation in an attempt to increase the level of drug permeation.

Among the enhancers used, octanoic acid had the strongest enhancement effects.

Pranoprofen gel containing octanoic acid as an enhancer, reduced oedema size by approximately $73 \%$ compared to that of the control group.

Honokiol was loaded into PF-127 micelles.

This system could have great potential applications for transdermal delivery of hydrophobic drugs such as honokiol.

The results showed that incorporation of piroxicam in microemulsion formulas could lead to enhancement of piroxicam release profiles by promoting constant and regular in vitro release.

Considering the in vitro release results, rheological properties and shelf life, a HPMC gel base containing $0.5 \%$ piroxicam in a microemulsion 
different gel bases such as, methyl cellulose, carboxy methyl cellulose, hydroxypropyl methyl cellulose, Carbopol 934, Carbopol 940, and PF-127 bases.

PF-127 gel formulated with terbinafine $\mathrm{HCl}$

Alginate and PF-127 used to design thermogels by either physical blending $(\mathrm{A}+\mathrm{P})$ or chemical grafting (AP).

Poloxamer/chitosan as vehicles for enhanced corneal permeation and sustained release of fluconazole.

PF-127 gel formulated with Ceragenin CSA-13 (synthetic mimic of cationic antibacterial peptides).

Topical HDL administration using 20\% PF-127 gel (applied on the adventitial side of vein grafts). formula was the optimal preparation among the studied formulations.

PF-127-based thermogelling formulation shows, to some extent, advantages as a vehicle for topical administration of terbinafine.

The porosity of the $\mathrm{A}+\mathrm{P}$ structure was greater compared to that of the AP structure.

The results of skin permeation, across porcine skin and nude mouse skin, suggested that these thermogels could produce sustained selegiline release, with AP showing the most-sustained permeation.

AP hydrogels exhibited linear permeation properties in transdermal delivery of selegiline.

The in vitro release studies showed sustained release of fluconazole from the poloxamer/chitosan formulation.

The chitosan solutions alone showed the greatest ex vivo drug permeation; however, the poloxamer/chitosan formulation presented a similar in vivo performance to a chitosan solution at $1.0 \%$.

In the presence of PF-127, CSA-13 haemolytic activity was greatly reduced.

PF-127 decreases the haemolytic, but not antibacterial, activity of CSA-13.

Topical HDL administration on the adventitial side of vein grafts attenuates vein-graft atherosclerosis via increased incorporation of circulating progenitor cells in the endothelium, enhanced endothelial regeneration, and reduced intimal inflammation.

\section{PLURONIC LECITHIN ORGANOGEL (PLO)}

A hydrophilic gel is designated as a hydrogel or organogel depending on the nature of the liquid component: water in hydrogels and an organic solvent in organogels $(66,67)$. Organogels are a vehicle base for the delivery of drugs through dermal and transdermal routes $(67,68)$.

Pluronic lecithin organogel (PLO) is a microemulsion-based gel that has been effectively used by physicians and pharmacists to deliver hydrophilic and lipophilic drugs topically and transdermally across the stratum corneum (lecithin gels slightly disorganise the structure of the skin, and thus permit the permeation of various substances) (66-69). It is a thermodynamically stable, viscoelastic, and biocompatible gel composed of phospholipids (lecithin), an organic solvent, and a polar solvent $(66,68,70-72)$.

A PLO is an opaque, yellow preparation, composed of isopropyl palmitate (or, less commonly, isopropyl myristate), soya lecithin (a permeation enhancer as it increases the fluidity of the stratum corneum - it is used as dispersing, emulsifying, and stabilising agent), water (acts as a stabilising and structure-forming agent in the process of PLO formation - it is also used for solubilising the PF-127 and polar drugs) and PF127 (72-74).

PLO consists of an oil phase (lecithin dissolved in isopropyl palmitate in a 1:1 ratio) and an aqueous phase (aqueous solution of 20-30\% of PF-127). The oil phase is prepared by mixing lecithin and isopropyl palmitate or isopropyl myristate (acts as a non-oleaginous emollient with very good spreading ability and also used for solubilising the lecithin) and allowing the mixture to stand overnight to ensure complete dissolution $(72,75)$. The aqueous phase is prepared by adding PF-127 to ice-cold water, placing the mixture in a refrigerator and agitating periodically to ensure complete dissolution. Sorbic acid at $0.2 \%(\mathrm{w} / \mathrm{w})$ or potassium sorbate is often added to the two phases as preservatives. The oil phase is then mixed with the aqueous phase (chilled before mixing) using a high-shear mixing method (75). 
An important aspect is that the drug may be incorporated within PLO by either dispersing into prepared PLO or, more commonly, by dispersing in either the oil phase or the aqueous phase, depending on drug solubility, before mixing of the two phases. If the drug is lipophilic it is usually mixed with propylene glycol to form a paste, which is then mixed with the aqueous or oily phase (75).

Studies show that the increase in concentration of lecithin decreases the cumulative percentage of drug released, which might be due to the extensive formation of a network-like structure with very high viscosity (47).

\section{Applications of PLO in pharmaceutical formulations}

There are several drugs, or combination of drugs, that have been incorporated within PLO. Some examples are: hormones (estriol, estradiol, dehydroepiandrosterone, progesterone, testosterone), non-steroidal anti-inflammatory drugs (ketoprofen, piroxicam, diclofenac), selective serotonin reuptake inhibitors (fluoxetine, paroxetine), antipsychotic drugs (haloperidol, prochlorperazine), secretin, selegiline hydrochloride, levodopa, morphine, dexamethasone, calcium channel blockers (diltiazem, nifedipine), Humulin $\mathrm{N}$ insulin, clonidine with gabapentin and ketamine hydrochloride, cyclopenzaprine with lidocaine, and methimazole $(67,68,71,74-78)$.

\section{Topical and dermal applications}

The coexistence of organic and aqueous phases by means of a structurally well-defined micellar network of phospholipids, a large interfacial area, and the possibility to entrap solutes within the gel matrix, along with long-term stability, makes PLO gels useful for a variety of applications. The organised microstructural matrix, amphiphilicity, supersolubilising capacity and interaction of the biolipids with skin tissues, are some of the major promoting factors for an enhanced transport of drug molecules into or across the skin (74).

In vivo studies suggested that PLO gels were a good delivery vehicle for local action. PLO gels show excellent drug permeability by diffusion through the lipid intracellular matrix and by slight disorganisation of skin $(70,79)$.

Table 2 shows many examples of the use of Pluronic lecithin organogels (PLO) in pharmaceutical systems over time.

Table 2. Examples of the use of Pluronic lecithin organogels (PLO) in pharmaceutical systems.

Pharmaceutical Formulations Ketoprofen in PLO gel.

Topical formulation of $2 \%$ diclofenac in PLO in the treatment of chronic lateral epicondylitis.

\section{Results}

Administration of ketoprofen in PLO gel offered convenience, produced fewer side effects, and alleviated pain in a specific location.

When subjects used diclofenac PLO, pain was significantly less than that during the pre-treatment, wash-out, or placebo PLO periods.

Topical 2\% diclofenac in PLO appears to provide effective short-term reduction in elbow pain and wrist extensor weakness associated with chronic lateral epicondylitis.

Topical formulation of the 5$\mathrm{HT}_{3}$ receptor antagonist ondansetron in PLO.

PLO formulated with ketamine.

Topical formulation of $2 \%$ diclofenac in PLO in the treatment of pain associated with mild to moderate osteoarthritis of the knee.
Application of PLO reduced the pain, mechanical hyperalgesia and inflammatory flare induced by intradermally injected capsaicin in a dose-dependent manner.

Attenuation of nociceptive and inflammatory effects of intradermally injected capsaicin.

Developed a PLO gel with ketamine, that when applied directly to the site of pain alleviates the sympathetic or neuropathic pain, while also avoiding the side effects associated with the drug.

Topical formulation of $2 \%$ diclofenac in a PLO appears to have therapeutic value in patients with mild to moderate osteoarthritis of the knee.

Patients experienced significantly less pain and stiffness when using diclofenac in PLO.

References 
Efficacy of PLO gels for topical use of local anaesthetics and nonsteroidal anti-inflammatory drugs

(NSAIDs)

Transdermal formulation testosterone in a PLO gel.

In vivo study of a single topical dose of ketoprofen $20 \%$ in a PLO.

In vivo study to determine the bioavailability of promethazine in a topical PLO.

PLO as a base for the delivery of bioactive polyunsaturated fatty acids from fish oil, eicosapentaenoic acid (EPA), docosahexaenoic acid (DHA) and ketoprofen.

In vivo study using $1 \mathrm{~mL}$ of morphine compounded at $10 \mathrm{mg} / \mathrm{mL}$ in PLO (randomised, placebo-controlled, double-blind, crossover study of five volunteers).

Topical formulation of ketamine hydrochloride (10\%) in PLO.

Preparation, characterisation, and in vitro release of a topical formulation of methimazole in PLO.

PLO formulated with flurbiprofen (prepared using PF-127, lecithin, flurbiprofen, isopropyl palmitate, water, sorbic acid, and potassium sorbate).

In vivo study using methadone (MTD) in PLO applied topically.

The objective of this study was to compare serum MTD levels achieved after topical and oral administration to
It is inferred that for those orofacial disorders that are regional, near the surface, and chronic, PLOs are more advantageous over systemic administration of drugs because of the rapid onset of action with a low side-effect profile.

Plasma concentrations of testosterone increased after 20-30 days and reached an apparent steady state during the administration period.

The relative bioavailability of ketoprofen was low and highly variable when the drug was administered as a single dose in a PLO-based ketoprofen $20 \%$ gel.

The topical promethazine, when applied in a PLO, was absorbed systemically and had serum concentrations much lower than after parenteral administration, with an absolute bioavailability of $2 \%$.

PLO was adapted to contain fish oil, ketoprofen, or both, with 1,8-cineole as penetration enhancer, and used to determine the in vitro permeation from infinite and finite dosing protocols across full-thickness porcine skin.

A PLO-based gel is capable of delivering EPA and DHA via a repeat finite dosing regimen, although there is evidence for the retention of these very lipophilic molecules within the gel matrix. Although to a lesser extent than EPA and DHA, ketoprofen was also substantially retained.

As morphine was seldom detected in plasma samples after topical administration and was unquantifiable when it was, the bioavailability of topical morphine was unquantifiable.

The results suggest that topical administration of morphine compounded in a PLO base for transdermal drug delivery is unlikely to provide relief of cancer-related pain.

In several patients, ketamine treatment of the symptomatic limb inhibited allodynia due to brushing the ipsilateral forehead, suggesting that the mechanism that mediates allodynia in the symptomatic limb contributed to allodynia at more remote sites.

The shows promise for the use of topical ketamine as opposed to parenteral and oral forms which often result in undesirable side effects.

Studies of release in vitro were carried out showing that the selected excipients do not pose an obstacle to the cession of methimazole.

Stability studies and freeze-thaw thermal cyclic tests were carried out, showing no phase separation of gel, and thus good gel stability.

The formulation showed a statistically significant antiinflammatory activity and is a non-irritant to the skin.

The topical application of an MTD-PLO gel in doses $<45$ $\mathrm{mg}$ /day did not result in trough MTD serum concentrations associated with analgesia.

An observer placebo response may explain the perceived benefit of MTD applied topically as a PLO gel in doses $<45$ 
hospice patients.

In vivo study using lorazepam, diphenhydramine, and haloperidol $(\mathrm{ABH})$ in PLO applied topically. $\mathrm{mg} /$ day. The evaluation of systemic absorption of MTDPLO gel in doses $>45 \mathrm{mg} /$ day is warranted.

No lorazepam or haloperidol was detected in any sample from any of the 10 volunteers down to a level of 0.05 $\mathrm{ng} / \mathrm{mL}$.

Diphenhydramine was found in multiple plasma samples at concentrations $>0.05 \mathrm{ng} / \mathrm{mL}$ in three patients, with the highest concentration of $0.30 \mathrm{ng} / \mathrm{mL}$ in one person at 240 minutes.

Overall, five out of ten patients exhibited detectable diphenhydramine in one or more samples, supporting limited absorption.

As commonly used, none of the lorazepam, haloperidol, or diphenhydramine in $\mathrm{ABH}$ gel is absorbed in sufficient quantities to be effective in the treatment of nausea and vomiting.

\section{CONCLUSIONS}

PF-127 and PLO gels present unique characteristics including their gelation behaviour and micellar properties. These characteristics make these polymers excellent carriers with the ability to administer an efficient concentration of drugs at the right time and location, reducing the adverse systemic reactions and increasing the patient's adherence to the therapeutic regimen, thereby also allowing for a reduction in the drug dose and, consequently, the costs.

These polymeric systems have the advantage of being thermodynamically stable, viscoelastic, biocompatible, and have specific and localised actions, thus increasing the potential effectiveness of the drugs.

PF-127 and PLO gels appear to be effective alternative vehicles for delivering drugs through topical and transdermal routes as these systems exhibit excellent drug permeability via diffusion through the lipid intracellular matrix and by slight disorganisation of skin.

\section{REFERENCES}

1. Gupta P, Vermani K, Garg S. Hidrigels: from controlled release to $\mathrm{pH}$-responsive drug delivery. Drug Discov Today, 2002; 7(10):569-579.

2. Jeong B, Gutowska A. Lessons from Nature: stimuli-responsive polymers and their biomedical applications. Trends Biotechnol, 2002; 20(7):305310 .

3. Kumar A, Srivastata A, Galaev IY, Mattiasson B. Smart polymers: physical forms and bioengineering applications. Prog Polym Sci, 2007; 32(10):1205-1237.

4. Stuart MAC, Huck WTS, Genzer J, Muiller M, Ober C, Stamm M, Sukhorukov GB, Szleifer I, Tsukruk VV, Urban M, Winnik F, Zauscher S,
Luzinov I, Minko S. Emerging applications of stimuli-responsive polymer materials. Nat Mater, 2010; 9(2):101-113.

5. Grainger, S.T.; El-Sayed, M.E.H., Stimulisensitive particles for drug delivery. biologicallyresponsive hybrid biomaterials: a reference for material scientists and bioengineers. World Scientific Publishing Co. Pte. Ltd., Danvers, pp 171-189, 2010.

6. Kuckling, D.; Urban, M.W., Handbook of stimuliresponsive materials. WILEY-VCH Verlag GmbH\& Co. KGaA, Weinheim, pp 1-26, 2011.

7. Almeida H, Amaral MH, Lobão P. Temperature and $\mathrm{pH}$ stimuli-responsive polymers and their applications in controlled and self-regulated drug delivery. J App Pharm Sci, 2012; 2(6):1-10.

8. Al-Tahami K, Singh J. Smart polymer based delivery systems for peptides and proteins. Recent Pat Drug Deliv Formul, 2007; 1(1): 65-71.

9. Nirmal HB, Bakliwal SR, Pawar SP. In-Situ gel: New trends in Controlled and Sustained Drug Delivery System. Int J PharmTech Res, 2010; 2(2): 1398-1408.

10. Abdel-Mottaleb MMA, Mortada ND, Elshamy AA, Awad GAS, Preparation and evaluation of Fluconazole Gels, Egypt. J Biomed Sci, 2007; 23(1) 266-286.

11. Escobar-Chávez JJ, López-Cervantes M, Naik A, Kalia YN, Quintanar-Guerrero D, GanemQuintanar A. Applications of thermo-reversible Pluronic F-127 gels in pharmaceutical formulations. J Pharm Pharm Sci, 2006; 9(3):339358.

12. Almeida, H.E.P.P.J., Polímeros inteligentes: classificação e aplicação em sistemas de libertação modificada de fármacos (Seminário de Doutoramento em Ciências Farmacêuticas). Porto: Faculdade de Farmácia da Universidade do Porto; 2012.

13. Dumortier G, Grossiord JL, Agnely F, Chaumeil JC. A review of polaxamer 407 pharmaceutical and pharmacological characteristics. Pharm Res, 2006; 23(12): 2709-2728. 
14. Gil ES, Hudson SM. Stimuli-responsive polymers and their bioconjugates. Prog Polym. Sci, 2004; 29(12): 1173-1222.

15. Cabana A, Ait-Kadi A, Juhász J. Study of the gelation process of polyethylene oxidepolypropylene oxide - polyethylene oxide copolymer (poloxamer 407) aqueous solutions. J Colloid Interface Sci, 1997; 190(2):307-312.

16. Veyries ML, Couarruze G, Geiger S, Agnely F, Massias L, Kunzli B, Faurisson F, Rouveix B. Controlled release of vancomycin from poloxamer 407 gels. Int J Pharm, 1999; 192(2):183-193.

17. Ricci EJ, Lunardi LO, Nanclares DMA, Marchetti JM. Sustained release of lidocaine from Poloxamer 407 gels. Int J Pharm, 2005; 288(2):235-244.

18. Fogueri LR, Singh S. Smart Polymers for Controlled Delivery of Proteins and Peptides: A Review of Patents. Recent Pat Drug Deliv Formul, 2009 Jan; 3(1): 40-48.

19. Aguilar MR, Elvira C, Gallardo A, Vázquez B, Román JS. Smart polymers and their applications as biomaterials. Available at: http://www.oulu.fi/spareparts/ebook_topics_in_t_e _vol3/abstracts/aguilar_01.pdf Accessed on: 02 out. 2011.

20. Bajpai AK, Shukla SK, Bhanu S, Kankane S. Responsive polymers in controlled drug delivery. Prog Polym Sci, 2008; 33(11): 1088-1118.

21. Hosseinzadeh H, Ataybi F, Dinarvand R, Ostad $\mathrm{SN}$, Chitosan-Pluronic nanoparticles as oral delivery of anticancer gemcitabine:preparation and in vitro study. Int J Nanomed, 2012; 7(1): 18511863.

22. Desai D, Zia H, Quadir A. Evaluation of selected micronized poloxamers as tablet lubricants. BASF Corporation. Available at: http://www.basf.com. Accessed on: 08 August 2012.

23. Ruchatz F, Lang S, Reich H, Fussnegger B. The combination of pluronic F127 and pluronic F68 - a new perspective for thermogelling drug drug delivery formulations. BASF Corporation. Available at: http://www.basf.com. Accessed on: 08 August 2012.

24. Schmolka IR. Artificial Skin I. Preparation and properties of Pluronic F-127 gels for treatment of burns. J Biomed Mater Res, 1972; 6(6):571-582.

25. Ribeiro, A.M., Preparação, Caracterização e Aplicação de Complexos de Inclusão de Miconazol com Metil- $\beta$-ciclodextrina: Administração Bucal (Master Tesis). Porto: Faculdade de Farmácia da Universidade do Porto; 2008.

26. Kulkarni SS, Aloorkar NH. Smart polymers in drug delivery: an overview. J Pharm Res, 2010; 3(1):100-108.

27. Lee SH, Lee JE, Baek WY, Lim JO. Regional delivery of vancomycin using pluronic F-127 to inhibit methicillin resistant Staphylococcus aureus (MRSA) growth in chronic otitis media in vitro and in vivo. J Control Release, 2004; 96 (1):1-7.

28. Feng Y, Gordts SC, Chen F, Hu Y, Craeyveld EV, Jacobs F, Carlier V, Feng Y, Zhang Z, Xu Q,
Ni Y, Geest B. Topical HDL administration reduces vein graft atherosclerosis in apo $\mathrm{E}$ deficient mice. Atherosclerosis, 2011; 214(2): 271-278.

29. Leszczyńka K, Namiot A, Cruz K, Byfield FJ, Won E, Mendez G, Sokolowski W, Savage PB, Bucki R, Janmey PA. Potential of ceragenin CSA13 and its mixture with pluronic F127 as treatment of topical bacterial infections. J Appl Microbiol, 2011; 110(1): 229-238.

30. El-Kamel AH. In vitro an in vivo evaluation of Pluronic F127-based ocular delivery system for timolol maleate. Int J Pharm, 2002; 241(1): 47-55.

31. Müller-Goymann L, Müller-Goymann CC. Preparation, Characterization, and In Vitro Permeation Study of Terbinafine $\mathrm{HCl}$ in Poloxamer 407-Based Thermogelling Formulation for Topical Application. AAPS PharmSciTech, 2011; 12(2):496-506.

32. Chang JY, Oh YK, Choi HG, Kim YB, Kim CK. Rheological evaluation of thermosensitive and mucoadhesive vaginals gels in physiological conditions. Int J Pharm, 2002; 241(1):155-163.

33. Ye F, Yaghmur A, Jensen H, Larsen SW, Larsen C, Østergaard J. Real-time UV imaging of drug diffusion and release from Pluronic F127 hydrogels. Eur J Pharm Sci, 2011; 43(4):236-243.

34. Pandit NK, Kisaka J. Loss of gelation ability of Pluronic ${ }^{\circledR}$ F127 in the presence of some salts. Int J Pharm, 1996; 145(1-2):129-136.

35. Pandit NK, Wang D. Salts effects on the diffusion and release rate of propranolol from poloxamer 407 gels. Int J Pharm, 1998; 167(1-2):183-189.

36. Sharma PK, Bhatia SR. Effect of antiinflammatories on pluronic F127: micellar assembly, gelation and partitioning. Int $\mathrm{J}$ Pharm, 2004; 278(2):361-377.

37. Cho CW, Choi JS, Shin SC. Development of the ambroxol gels for enhanced transdermal delivery. Drug Dev Ind Pharm, 2008; 34(3):330-335.

38. Choi JS, Shin SC. Preparation and evaluation of pranoprofen gel for percutaneous administration. Drug Dev Ind Pharm, 2007; 33(1):19-26.

39. Morishita M, Barichello JM, Takayama K, Chiba Y, Tokiwa S, Nagai T. Pluronic F-127 gels incorporating highly purified unsaturated fatty acids for buccal delivery of insulin. Int J Pharm, 2001; 212(2):289-293.

40. Bentley M, Marchetti J M, Nágila R, Ali-Abi Z, Collet JH. Influence of lecithin on some physical chemical properties of poloxamer gels: rheological, microscopic and in vitro permeation studies. Int J Pharm, 1999; 193(1):49-55.

41. Gonjari ID, Karmarkar AB, Kasture PV. In vitro evaluation of different transnasal formulations of sumatriptan succinate: A comparative analysis. Drug Discov Ther, 2009; 3(6): 266-271.

42. Fitzgerald RR, Walters JD. Accumulation of topical naproxen by cultures oral epithelium. J Dent Res, 2007; 86(8): 775-779.

43. Yuan Y, Ying C, Li Z, Hui-ping Z, Yi-Sha G, Bo $Z$, Xia H, Ling Z, Xiao-hui W, Li C. Thermosensitive and mucoadhesive in situ gel 
based on poloxamer as new carrier for rectal administration of nimesulide. Int $\mathrm{J}$ Pharm, 2012; 430(1-2): 114-119.

44. Shin SC, Kim J. Enhanced permeation of triamcinolone acetonide through the bucal mucosa. Eur J Pharm Biopharm, 2000; 50(2): 217220.

45. Barichello JM, Morishita M, Takayama K, Nagai T. Absorption of insulin from Pluronic F-127 gels following subcutaneous administration in rats. Int J Pharm, 1999; 184(2):189-199.

46. Ruel-Gariépy E, Leroux J. In situ-forming hydrogels-review of temperature-sensitive systems. Eur J Pharm Biopharm, 2004; 58(2):409426.

47. Pandey MS, Belgamwar VS, Surana SJ. Topical Delivery of Flurbiprofen from Pluronic Lecithin Organogel. Indian J Pharm Sci, 2009; 71(1): 8790.

48. Miyazaki S, Takeuchi S, Yokouchi C, Takada M. PLuronic F-127 gels as a vehicle for topical administration to anticancer agents. Chem Pharm Bull, 1984; 32(10):4205-4208.

49. Miyazaki S, Tobiyama T, Takada M, Attwood D. Percutaneous Absorption of Indomethacin from Pluronic F-127 Gels in Rats. J Pharm Pharmacol, 1995; 47(6):455-457.

50. Chi SCh, Do K, Tan HK, Chun HW. Antiinflammatory and analgesic transdermal gel, United States Patents. Patent number 5,527,832, 1996.

51. Liaw J, Lin YCh. Evaluation of poly(ethylene oxide)-poly(propylene oxide)- poly(ethylene oxide) (PEO-PPO-PEO) gels as a release vehicle for percutaneous fentanyl. J Control Rel, 2000; 68(2):273-282.

52. Erukova VY, Krylova OO, Antonenko YN, MelikNubarov NS. Effect of ethylene oxide and propylene oxide block copolymers on the permeability of bilayer lipid membranes to small solutes including doxorubicin. Biochim Biophys Acta, 2000; 1468(1- 2):73-86.

53. Shin S, Cho C, Oh I. Enhanced efficacy by percutaneous absorption of piroxicam from the poloxamer gel in rats. Int J Pharm, 2000; 193(2): 213-218.

54. Shin SC, Cho CW, Oh IJ. Effects of non ionic surfactants as permeation enhancers towards piroxicam from the poloxamer gel through rat skins. Int J Pharm, 2001; 222(2):199-203.

55. Wang YY, Hong CT, Chiu WT, Fang JY. In vitro and in vivo evaluations of topically applied capsaicin and nonivamide from hydrogels. Int $\mathrm{J}$ Pharm, 2001; 224(1-2): 89-104.

56. Fang JY, Leu, YL, Wang YY, Tsai YH. In vitro topical application and in vivo phramacodynamic evaluation of nonivamide hydrogels using Wistar rat as an animal model. Eur J Pharm Sci, 2002; 15(5):417- 423 .

57. Zhang L, Parson DL, Navarre C, Kompella UB. Development and in-vitro evaluation of sustained release poloxamer 407 (P407) gel formulations of ceftiofur. J Control Release, 2002; 85(1-3):73-81.
58. Shawesh A, Kallioinen S, Hellen L, Yliruusi J. Pluronic F-127 gels as a vehicle for topical formulations of indomethacin and rheological behaviour of these formulations. Pharmazie, 2002; 57(3):186- 190.

59. Pillai O, Pachagnula R. Transdermal delivery of insulin from poloxamer gel: ex vivo and in vivo skin permeation studies in rat using iontophoresis and chemical enhancers, J Control Release, 2003; 89(1):127-140.

60. Stamatialis DF, Rolevink HHM, Koops GH. Transdermal timolol delivery from a Pluronic gel. J Control Release, 2006; 116(2):e53-e55.

61. Shin SC, Cho CW. Enhanced transdermal delivery of pranoprofen from the bioadhesive gels. Arch Pharm Res, 2006; 29(10):928-933.

62. Zheng X, Wang X, Gou M, Zhang J, Men K, Chen L, Luo F, Zhao X, Wei Y, Qian Z. A novel transdermal honokiol formulation based on Pluronic F127 copolymer. Drug Deliv, 2010; 17(3):138-144.

63. Abd-Allah FI, Dawaba HM, Ahmed AM. Preparation, characterization, and stability studies of piroxicam-loaded microemulsions in topical formulations. Drug Discov Ther, 2010; 4(4):267275.

64. Chen CC, Fang CL, Al-Suwayeh SA, Leu YL, Fang JY. Transdermal delivery of selegiline from alginate-Pluronic composite thermogels. Int $\mathrm{J}$ Pharm, 2011; 415(1-2):119-128.

65. Gratieri T, Gelfuso GM, de Freitas O, Rocha EM, Lopez RF. Enhancing and sustaining the topical ocular delivery of fluconazole using chitosan solution and poloxamer/chitosan in situ forming gel. Eur J Pharm Biopharm, 2011; 79(2):320-327.

66. Agrawal V, Gupta V, Ramteke S, Trivedi P. Preparation and Evaluation of Tubular Micelles of Pluronic Lecithin Organogel for Transdermal Delivery of Sumatropin. AAPS Pharm SciTech, 2010; 11(4): 1718-1725.

67. Patil KD. Organogel: topical and transdermal drug delivery system. Int J Pharm Res Dev, 2011; 3(6): 58-66.

68. Williman H, Walde P, Luisi PL, Gazzaniga A, Stroppolo F. Lecithin organogel as matrix for transdermal transport of drugs. J Pharm Sci, 1992; 81(9):871-874.

69. Finch PM, Knudsen L, Drummond PD. Reduction of allodynia in patients with complex regional pain syndrome: A double-blind placebo-controlled trial of topical Ketamine. Pain, 2009; 146(1-2): 18-25.

70. Loyd A. The history of pluronic lecithin organogel: An interview with Marty Jones. Int J Pharma Comp, 2003; 7:180-2.

71. Ruiz MA, Clares B, Morales ME, Gallardo V. Preparation, rheological study, and characterization of an organogel as a system for transdermal release of active principles. Pharm Dev Technol, 2007; 12(6): 637-644.

72. Belgamwar VS, Pandey MS, Chauk DS, Surana SJ. Pluronic lecithin organogel. Asian J of Pharm, 2008; 2(3): 134-138. 
73. Murdan S. Organogels in drug delivery. Expert Opin Drug Deliv, 2005; 2(3): 489-505.

74. Kumar R, Katare OP. Lecithin organogels as a potential phospholipid-structured system for topical drug delivery: A review. AAPS PharmaSciTech, 2005; 6(2):298-310.

75. Murdan S. A review of pluronic lecithin organogel as a topical and transdermal drug delivery system. Hospital Pharmacist, 2005; 12(7): 267-270.

76. Franckum J. Ramsay D, Das NG, Das SK. Pluronic lecithin organogel for local delivery of anti-inflammatory drugs. Int $\mathrm{J}$ Pharma Comp, 2004; 8(1): 101-105.

77. Stanos SP. Topical Agents for the management of musculoskeletal pain. J Pain Symptom Manage, 2007; 33(3): 342-355.

78. Gupta H, Sharma A, Shrivastava B. Pluronic and Chitosan based In situ gel system for periodontal application. Asian J Pharm, 2009; 3(2):94-96.

79. Trepanier LA. Medical Management of Hyperthyroidism. Clin Tech Small Anim. Pract, 2006; 21(1):22-28.

80. Berti JJ, Lipskys JJ. Transcutaneous drug delivery: a practical review. Mayo Clin Proc, 1995; 70(6):581-586.

81. Burnham R, Gregg R, Healy S, Steadwars R. The effectiveness of topical diclofenac for lateral epicondylitis. Clin J Sport Med, 1998; 8(2): 78-81.

82. Giordano J, Daleo C, Sacks SM. Topical ondansetron attenuates nociceptive and inflammatory effects of intradermal capsaicin in humans. Eur J Pharmacol, 1998; 354(1):R13-R14.

83. Flores JA, Crowley KL, inventors. Process for the preparation of ketamine ointment. US patent 5817 699. October 6, 1998.

84. Grace D, Rogers J, Skeith K, Anderson K. Topical diclofenac versus placebo: a double blind, randomised clinical trial in patients with osteoarthritis of the knee. J Rheumat, 1999; 26(12):2659:2663.
85. Padilla M, Clark GT, Merill RL. Topical medications for orofacial pain: a review. J Am Dent Assoc, 2000; 131(2):184-195.

86. Kryger A, inventor. Topical testosterone formulations. PCT Int Appl WO2002055020. July 18, 2002.

87. Dowling TC, Arjomand M, Lin ET, Allen LV Jr, McPherson ML. Relative bioavailability of Ketoprofen $20 \%$ in a poloxamer-lecithin organogel. Am J Health Syst Pharm, 2004; 61(23): 2541-2544.

88. Glisson JK, Wood RL, Kyle PB, Cleary JD. Bioavailability of promethazine in a topical pluronic lecithin organogel: A pilot study. Int J Pharma Comp, 2005; 9:242-246.

89. Richards H, Thomas CP, Bowen JL, Heard CM. In vitro transcutaneous delivery of ketoprofen and polyunsaturated fatty acids from a pluronic lecithin organogel vehicle containing fish oil. J Pharm Pharmacol, 2006; 58(7):903-908.

90. Paice JA, Von Roenn JH, Hudgins JC, Luong L, Krejcie TM, Avram MJ. Morphine Bioavailability from a Topical Gel Formulation in Volunteers. J Pain Symptom Manage, 2008; 35(3): 314-320.

91. Morales ME, Clarés B, López-Viota M, Ruiz MA. Preparation, characterization and in vitro release of new transdermal methimazole as alternative to oral delivery. Drug Deliv, 2009; 16(1): 1-10.

92. Pandey M, Belgamwar V, Gattani S, Surana S, Tekade A. Pluronic lecithin organogel as a topical drug delivery system. Drug Deliv, 2010; 17(1):3847.

93. Sylvester RK, Schauer C, Thomas J, Steen P, Weisenberger A. Evaluation of Methadone Absorption After Topical Administration to Hospice Patients. J Pain Symptom Manage, 2011; 41(5): 828-835.

94. Smith TJ, Ritter JK, Poklis JL, Fletcher D, Coyne PJ, Dodson P, Parker G. ABH Gel Is Not Absorbed From the Skin of Normal Volunteers. J Pain Symptom Manage, 2012; 43(5): 961-966. 